\title{
Novel Artificial Biofilm Equivalent for Denture Surfaces: A Pilot Study
}

\author{
Jan-Philipp Kunze (D), Klaus Boening (D), Heike Meissner (D), \\ and Katarzyna Kresse-Walczak
}

\begin{abstract}
Department of Prosthetic Dentistry, Carl Gustav Carus Faculty of Medicine, Technische Universität Dresden, Fetscherstr. 74, 01307 Dresden, Germany
\end{abstract}

Correspondence should be addressed to Katarzyna Kresse-Walczak; katarzyna.kresse@uniklinikum-dresden.de

Received 2 December 2021; Revised 4 January 2022; Accepted 5 January 2022; Published 27 January 2022

Academic Editor: Yulin Deng

Copyright (c) 2022 Jan-Philipp Kunze et al. This is an open access article distributed under the Creative Commons Attribution License, which permits unrestricted use, distribution, and reproduction in any medium, provided the original work is properly cited.

\begin{abstract}
The microbial population of the dental biofilm is embedded in an extracellular matrix that contains organic polymers such as polysaccharides. The extracellular matrix promotes biofilm adhesion on surfaces of dental prostheses and acts as a protective barrier. Thus, a breakdown of the extracellular matrix is crucial for an effective mechanical biofilm removal by brushing. The purpose of this study was to develop an artificial biofilm equivalent (ABE) that is able to mimic the mechanical properties of a natural biofilm concerning abrasion resistance. It contains the two polysaccharides chitosan (ChS) and methylcellulose (MC). Polymethylmethacrylate (PMMA) cylinders $(n=80)$ were manufactured and coated with the ABE with varying concentration ratios of $\mathrm{ChS}$ and MC. Eight test series $(n=8$ each) with different mixing ratios of ChS/MC were tested for their abrasion resistance to brushing in a toothbrush simulator. For the ABE, a total of $2.0 \mathrm{~g}$ of polysaccharides were added to $100 \mathrm{ml}$ of $2 \%$ acetic acid: $0.3-1.0 \mathrm{~g}$ ChS and 1.0-1.7 MC, respectively. Furthermore, two control series $(n=8$ each) with $2.0 \mathrm{~g}$ of ChS only or $2.0 \mathrm{~g}$ of $\mathrm{MC}$ only were performed. Coated specimens were subjected to an increasing number of brushing strokes from 5 to 45 via abrasion test. The specimens were photographed, and a computerized planimetric method $(\mathrm{CPM})$ was used to calculate the percentage of remaining $\mathrm{ABE}$ on the brushed areas of the PMMA cylinders. The abrasion resistance of the $\mathrm{ABE}$ to brushing decreased with an increasing ratio of $\mathrm{MC}$ in the mixture. The abrasion resistance of the $\mathrm{ABE}$ can be adjusted by changing the ratio of $\mathrm{ChS}$ and $\mathrm{MC}$.
\end{abstract}

\section{Introduction}

Oral hygiene and dental care have changed over the years with more aging people retaining their natural teeth [1]. The current German Oral Health Study (DMS V) shows significant improvement in oral health and describes a shift in tooth loss towards older age [2]. Nevertheless, the number of people wearing partial or complete removable dentures is still substantial on a global scale and the number of geriatric patients is expected to increase over the next years [3,4]. Denture-induced chronic oral mucosal disorders such as denture stomatitis have a high prevalence and are often associated with inadequate biofilm removal from denture sur- faces and Candida albicans infections [3, 5, 6]. Thus, within the multifactorial pathogenesis of denture stomatitis, poor denture hygiene is considered the key factor [3]. This may explain why denture stomatitis is present particularly in people with reduced general conditions and a low ability to clean their dentures sufficiently and regularly and underlines the importance of biofilm removal for maintaining oral and general health [7-9].

The dental biofilm forms a three-dimensional structure of microorganisms, embedded in a self-produced matrix, which consists of extracellular polymeric substances (EPS), such as polysaccharides as glucans, proteins, and lipids [10, 11]. The biofilm matrix provides among others mechanical 
stability and adhesion to surfaces and acts as a protective barrier $[11,12]$.

The literature describes a variety of chemical agents to simulate the dental biofilm. There are poly methyl vinyl ether/maleic anhydride (PVM/MA) copolymer, or various types of paints which are used mainly for evaluation of different toothbrushes designs $[13,14]$, or dental adhesive stabilizing agent with ground chalk for denture care training [15]. However, these agents are often water-soluble or do not adhere sufficiently to the surface. Furthermore, a standardized texture and application method as required in research is difficult to obtain from these equivalents. Other methods to simulate the dental biofilm focus more on microbial models [16-18]. They may be closer to the clinical conditions but are more complex, costly, and less effective when investigating mechanical biofilm removal and not appropriate for denture care training. There seems to be a lack of suitable artificial biofilm equivalents, which show adhesion and abrasion resistance comparable to natural biofilm.

ChS films on denture materials such as PMMA show good adhesion and high abrasion resistance that can withstand up to 30.000 brushing cycles in a toothbrush simulator [19]. This feature predisposes ChS as a suitable substance to develop an ABE. However, the abrasion resistance to brushing of pure ChS films is far too high. The addition of MC to the ChS film may allow a controlled reduction of the abrasion resistance and hence an adjustment of this key property to that of a natural denture biofilm. Therefore, the $\mathrm{ABE}$ in this study is composed of the two polysaccharides ChS and MC.

The biopolymer ChS contains (1-4)-2-acetamino-2deoxy- $\beta$-D-glucans and is obtained by $\mathrm{N}$-deacetylation of chitin mainly from crabs and shrimps [20]. ChS is a biomaterial of high attention mostly because of its biocompatibility, nontoxicity, and wide range of possible applications, for example as a bioadhesive and as an antimicrobial material [21]. MC is a methyl ether of cellulose and is produced by treating cellulose fibers with lye and methylating the alkaline cellulose with methyl chloride [22]. MC has an amphiphilic character and is used for instance as a stabilizer in emulsions and to maintain the viscosity of solutions [23, 24]. MC also has excellent film-forming properties [25].

ChS consists of $\beta$-1,4-glycosidically linked $\mathrm{N}$ acetylglucosamine residues. It dissolves in organic acidic solutions such as acetic acid due to the protonation of the amino groups. The free amino groups allow chemical interactions with other polymers such as MC [20]. In acetic acid solutions of $\mathrm{ChS}$ and $\mathrm{MC}$, chemical bonds are formed between the positively charged amino groups $(-\mathrm{NH} 3+)$ of $\mathrm{ChS}$ and the partially negatively charged hydroxyl groups $(-\mathrm{OH})$ of $\mathrm{MC}[22,26,27]$.

It was the purpose of this study to develop an $\mathrm{ABE}$ based on $\mathrm{ChS}$ and MC. The ABE to be developed should be able to mimic the mechanical properties of a natural biofilm concerning abrasion resistance. The ABE should allow the adjustment of its mechanical properties for a close simulation of the consistencies of natural biofilms. The aim was to analyze how abrasion resistance can be controlled by the addition of specific amounts of MC to ChS and thus evaluate the feasibility of such ChS/MC films as ABE.

\section{Materials and Methods}

2.1. Preparation of the $A B E$. For the production of the $A B E$, the following ingredients were used: ChS (Chitoscience Chitosan 90/500, MW 200-400 kDa, DD 87.6\%-92.5\%, Heppe Medical Chitosan GmbH, Halle, Germany), 2\% acetic acid ( $\mathrm{pH}$ 2.8, pharmacy of University Hospital C.G. Carus, Dresden, Germany), and MC (400 cP, Sigma-Aldrich Co., St. Louis, USA).

ABE was prepared in eight different formulations (test series 1 to 8, Table 1). Each ABE formulation was prepared as follows: first $\mathrm{ChS}$ was dissolved in $100 \mathrm{ml}$ acetic acid $(2 \% \mathrm{w} / \mathrm{w})$ solution at $60^{\circ} \mathrm{C}$ by stirring for $2 \mathrm{~h}$ using a magnetic stirrer (RET control-visc, IKA ${ }^{\circledR}$-company, Staufen, Germany). After $2 \mathrm{~h}$, the corresponding amount of $\mathrm{MC}$ was added at $60^{\circ} \mathrm{C}$ initial temperature and the solution was stirred for $4 \mathrm{~h}$ and simultaneously cooled down to room temperature. Additionally, two control groups with $2.0 \mathrm{~g}$ ChS only and $2.0 \mathrm{~g}$ MC only were prepared.

2.2. Specimen Preparation. Cylindrical PMMA specimens $(\varnothing=12.75 \mathrm{~mm}$, height $=6 \mathrm{~mm}$, Palapress clear, Heraeus, Hanau, Germany) were produced according to manufacturer's instructions as follows: powder to liquid ratio $10 \mathrm{~g}$ to $7 \mathrm{ml}$, mixing time $15 \mathrm{~s}$, and polymerization time $20 \mathrm{~min}$ at $55^{\circ} \mathrm{C}$ at 2.0 bar (Palamat elite, Kulzer $\mathrm{GmbH}$, Hanau, Germany). The specimens were ultrasonically cleaned in distilled water at room temperature $\left(23^{\circ} \mathrm{C}\right)$ for $10 \mathrm{~min}$, and the test surfaces of the specimens were sandblasted with silica-modified aluminum oxide (Rocatec Plus, $3 \mathrm{M}$ Espe $\mathrm{GmbH}$, Seefeld, Germany) at a jet angulation of $90^{\circ}$ and a distance of $3 \mathrm{~cm}$ at 2.8 bar blast pressure for $10 \mathrm{~s}$. A standardized spacer was used to keep a constant $3 \mathrm{~cm}$ distance.

For each test series, eight specimens were covered with $100 \mu \mathrm{l}$ of $\mathrm{ABE}$, using a pipette (Eppendorf Research blue 100-1000 $\mu$ l Eppendorf AG, Hamburg, Germany) with shortened tips (reduced to $10 \mathrm{~mm}$ length) to enlarge the tip opening. The applied $\mathrm{ABE}$ was dried for $16 \mathrm{~h}$ at $45^{\circ} \mathrm{C}$ (B6030 heating cabinet, Heraeus, Hanau, Germany) and then neutralized with $1 \mathrm{~mol}$ sodium hydroxide solution (NaOH, pharmacy of University Hospital C.G. Carus, Dresden, Germany) for $10 \mathrm{~min}$. Afterward, the specimens were rinsed in tap water and then stored in tap water for $30 \mathrm{~min}$.

2.3. Abrasion Tests. Subsequently the ABE were dyed with $3 \%$ erythrosine solution (Speikoplaque 3\%, SPEIKO ${ }^{\circledR}$-Dr. Speier GmbH, Bielefeld, Germany). Then, abrasion tests were carried out in an automatic toothbrushing simulator (Willytec GmbH, München, Germany). Toothbrushes (Elmex sensitive, GABA International, Therwil, Switzerland) with $300 \mathrm{~g}$ contact weight and tap water as the working medium were used. The specimen surfaces were brushed with a number of $5,10,15,25$, and 45 brushing strokes (one stroke $=$ one forward and backward movement). Brushing strokes were triangular (saw tooth) oscillations with movements of an amplitude of $10 \mathrm{~mm}$ along the $y$-axis at $2.5 \mathrm{~Hz}$ and slow horizontal movements of $0.25 \mathrm{~Hz}$ at an amplitude of $10 \mathrm{~mm}$ along the $x$-axis. This brushing stroke pattern is fixed within the mechanics of the toothbrush 
TABle 1: Test series with mixing ratios of ChS/MC in $100 \mathrm{ml}$ aqueous solution containing $2 \%$ acetic acid.

\begin{tabular}{ll}
\hline Test series & ABE-mixture (g) \\
\hline TS 1 & $0.3 \mathrm{ChS} / 1.7 \mathrm{MC}$ \\
TS 2 & $0.4 \mathrm{ChS} / 1.6 \mathrm{MC}$ \\
TS 3 & $0.5 \mathrm{ChS} / 1.5 \mathrm{MC}$ \\
TS 4 & $0.6 \mathrm{ChS} / 1.4 \mathrm{MC}$ \\
TS 5 & $0.7 \mathrm{ChS} / 1.3 \mathrm{MC}$ \\
TS 6 & $0.8 \mathrm{ChS} / 1.2 \mathrm{MC}$ \\
TS 7 & $0.9 \mathrm{ChS} / 1.1 \mathrm{MC}$ \\
TS 8 & $1.0 \mathrm{ChS} / 1.0 \mathrm{MC}$ \\
TS 9 (negative control) & $0.0 \mathrm{ChS} / 2.0 \mathrm{MC}$ \\
TS 10 (positive control) & $2.0 \mathrm{ChS} / 0.0 \mathrm{MC}$ \\
\hline
\end{tabular}

ABE: artificial biofilm equivalent; TS: test series; ChS: chitosan; MC: methylcellulose.

simulator and cannot be changed. The simulator always starts on the right side of the specimens. It moves first to the center (initial movement) then to the right, and afterward to the left across the specimen surface (brushing movement). When interrupted after only five brushing strokes only the right half of the specimen surface is brushed completely while the left half is brushed incompletely. Due to that inherent limitation of the toothbrush simulator a homogeneous brushing across the entire specimen surface was not possible at low brushing stroke numbers. Thus, in all experiments, only the right specimen half was evaluated for the remaining $\mathrm{ABE}$.

After brushing the surfaces were photographed (Canon EOS 600 D, Tokyo, Japan; Canon MACRO $0.3 \mathrm{~m} / 0.98 \mathrm{ft}$, $40 \mathrm{~mm}$, Tokyo, Japan; Yongnuo YN-14EX Macro Ring LITE Flash Light, Shenzhen, China) with the following camera settings: $1 / 200$, ISO $200, \mathrm{~F} 9.0$, and the amount of remaining $\mathrm{ABE}$ analyzed by means of CPM.

\subsection{Planimetric and Descriptive Analyses of Remaining ABE.} For the CPM, the software Adobe Photoshop CC 2017 (Adobe Systems Incorporated, San José, California, USA) was used. The analysis of remaining ABE on the specimen surfaces after brushing was carried out according to a standardized protocol published by Al Jaghsi et al. [28]. The image files were opened with Photoshop (layer 0). To calculate the percentage of remaining $\mathrm{ABE}$, the area of the test specimen surface to be analyzed was isolated digitally from the rest of the picture (layer 1). Using the Color Range Tool (settings: tolerance $=30$, range $=100 \%$ ), the color ranges corresponding to the dyed $\mathrm{ABE}$ were filtered and isolated into a new layer (layer 2).

The remaining $\mathrm{ABE}$ was calculated by the ratio of pixels of the two generated layers 1 (surface of the specimen half) and 2 (dyed ABE). Data were calculated and stored using Excel 2019 software (Microsoft Office, Microsoft Corporation, Redmond, WA, USA). SPSS software (IMB SPSS 25, IMB, Armonk, NY, USA for Windows) was used for descriptive analysis.

\section{Results}

The percentage of remaining $\mathrm{ABE}$ after specimens brushing decreased with an increase in the proportion of $\mathrm{MC}$ in the $\mathrm{ChS} / \mathrm{MC}$ mixture (Figure 1, Table 2). Also, the percentage of remaining $\mathrm{ABE}$ decreased with an increase in brushing stroke numbers (Figures 2(a)-2(e)). Test series with a low MC content (TS 5 to TS 7) showed a decrease in remaining $\mathrm{ABE}$ only after a higher number of brushing strokes (25 to 45 strokes). Test series with a high MC content (TS 1 and TS 2) showed little or no remaining ABE already after 10 to 15 brushing strokes.

In TS 8 and TS 10 (TS $10=$ no MC, ChS only, positive control), the $\mathrm{ABE}$ was still present across the entire evaluated surface after 45 brushing strokes. In the TS 9 (no ChS, $\mathrm{MC}$ only, negative control), no ABE was detectable even after storage in water and at the time of staining with erythrosine solution.

A dependence of abrasion resistance of the $\mathrm{ABE}$ to brushing strokes from the amount of added MC was shown. With increasing $\mathrm{MC}$ content and thus decreasing $\mathrm{ChS}$ content with the same total biopolymer quantity given, a decrease in abrasion resistance to brushing was verifiable.

\section{Discussion}

This pilot study described a novel $\mathrm{ABE}$ made from a mixture of acetic ChS solution with MC added. The ABE was applied to PMMA surfaces and formed an adhering film after drying in an oven and subsequent neutralization with $\mathrm{NaOH}$. Its abrasion resistance to brushing was investigated. ABE abrasion resistance to brushing depended on the ratio of the content of $\mathrm{ChS}$ and MC. An increase in the amount of MC and a corresponding decrease in the amount of $\mathrm{ChS}$ while keeping the total biopolymer quantity constant caused a decrease in abrasion resistance of the $\mathrm{ABE}$ and vice versa.

Natural biofilms are composed of up to $90 \%$ of EPS, which contain polysaccharides, proteins, and others. EPS influences the physiomechanical properties of biofilms such as adhesion to surfaces and biofilm stability that ensures protection $[11,29]$. These are important properties, which influence the abrasion resistance of oral biofilms to brushing [11].

Biofilm adhesion in the oral cavity is a complex process. After pellicle formation, the attachment of bacteria is mediated by adhesins [30]. The microorganisms multiply and embed themselves in an extracellular matrix that protects and stabilizes the growing biofilm $[10,11,30]$. Different local factors, such as $\mathrm{pH}$, oxygen supply, or electrostatic charges influence biofilm adhesion in the oral environment [30]. In the case of denture bases, the effect of biofilm formation and adhesion is further enhanced by surface roughness and the permanent contact to the alveolar mucosa [30]. Oral biofilm formation differs between individuals and even in different parts of the oral cavity [30].

The microorganism mass in the biofilm has less influence on abrasion resistance to bushing rather than the extracellular matrix [11]. Thus, it was the author's intention to develop an $\mathrm{ABE}$ that allows to mimic the abrasion resistance of the oral biofilm on denture bases in vitro. This novel ABE 


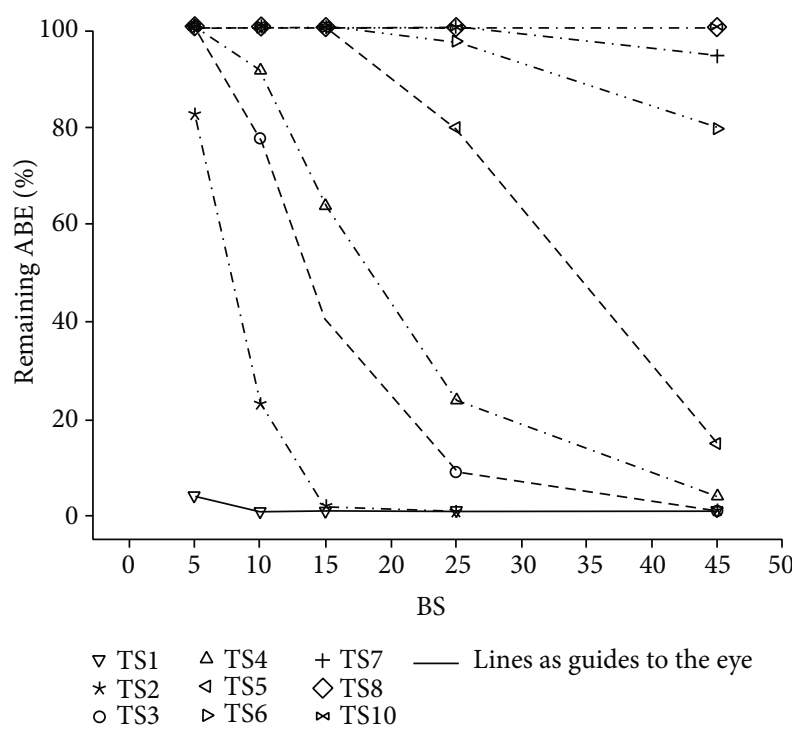

FIGURE 1: Influence of brushing strokes (BS) and mixing ratio on the percentage of remaining artificial biofilm equivalent $(\mathrm{ABE})$ in the test series TS 1 to TS 8 and control (TS 10). See also Table 2. The lines connecting data points are guides to the eye only.

TABle 2: Descriptive data (mean, brackets contain standard deviation) for the percentage of remaining artificial biofilm equivalent $(\mathrm{ABE})$ for test series 1 to 10 after brushing with 5 to 45 brushing strokes.

\begin{tabular}{lccccc}
\hline \multicolumn{5}{c}{ Remaining ABE (\%) } \\
& 5 BS & $10 \mathrm{BS}$ & $15 \mathrm{BS}$ & $25 \mathrm{BS}$ & $45 \mathrm{BS}$ \\
\hline TS 1 & $3(4)$ & $0(0)$ & $0(0)$ & $0(0)$ & $0(0)$ \\
TS 2 & $82(12)$ & $22(12)$ & $1(2)$ & $0(0)$ & $0(0)$ \\
TS 3 & $100(0)$ & $77(10)$ & $40(12)$ & $8(5)$ & $0(0)$ \\
TS 4 & $100(0)$ & $91(9)$ & $63(19)$ & $23(19)$ & $3(5)$ \\
TS 5 & $100(0)$ & $100(0)$ & $100(0)$ & $79(14)$ & $14(12)$ \\
TS 6 & $100(0)$ & $100(0)$ & $100(0)$ & $97(6)$ & $79(14)$ \\
TS 7 & $100(0)$ & $100(0)$ & $100(0)$ & $100(0)$ & $94(5)$ \\
TS 8 & $100(0)$ & $100(0)$ & $100(0)$ & $100(0)$ & $100(0)$ \\
TS 9 & $0(0)$ & $0(0)$ & $0(0)$ & $0(0)$ & $0(0)$ \\
TS 10 & $100(0)$ & $100(0)$ & $100(0)$ & $100(0)$ & $100(0)$ \\
\hline
\end{tabular}

ABE: artificial biofilm equivalent; TS: test series; BS: brushing stroke.

might be suitable to evaluate mechanical cleaning strategies and the effectiveness of cleaning equipment in research and education.

In vitro studies using mechanical brushing simulators are an important part of the evaluation of the effectiveness of oral hygiene measures such as toothbrushes long since [31]. However, suitable, validated substances to simulate the oral biofilm are missing.

Ledder et al. used an in vitro oral biofilm model to conduct toothbrush testing on typodont teeth [31]. This is comparable to our study only to a limited extent. Ledder et al. cultivated natural biofilms via salivary inoculate using a drip-flow model [31]. This approach needs the participation of volunteers and thus mostly ethical approval. Additionally, there is bias caused by the volunteers as the biofilm formation differs between individuals [30]. Our approach bypasses these hurdles. It is not a natural biofilm as described by Ledder et al. but intended to simulate similar abrasion resistance to brushing [31].

Available in vitro biofilm models are often limited by elaborate and costly laboratory methods and restricted by the number of microorganisms included. Especially, simplified one-species biofilm models cannot mimic the complexity of dental biofilms [11]. The oral cavity is colonized by up to 1000 microbial species [30]. Thus, the development of in vitro biofilms with similar quality to that in the oral cavity is costly, associated with complex laboratory techniques, problems of standardization, and reproducibility, or involves the active cooperation of volunteers $[32,33]$. Thus, simple artificial biofilm models prefer different substances such as paints when investigating the cleaning efficacy of toothbrushes [14]. However, these artificial biofilm models also have their limitations and might be considered oversimplified since they show either solubility in aqueous media, difficulties in standardization and reproducibility, or surface adhesion grossly diverging from a natural biofilm.

A PVM/MA copolymer based on ethyl ester alcohol was found to be promising to simulate the adhesion of the dental biofilm on typodont teeth [13]. Nevertheless, the adhesion to denture materials had not been investigated and therefore cannot be compared with this study.

Lang et al. used a "plaque-simulating substrate" for validation of robot simulation of tooth brushing. Nevertheless, the authors disclose no details about the composition of this substrate [34]. Thus, a comparison is not possible.

ChS can adhere to denture materials such as PMMA [19]. ChS and MC molecules interact physicochemically $[22,26,27]$. This might be a factor in the control of the abrasion resistance by the ratio of $\mathrm{ChS}$ and $\mathrm{MC}$ and predisposes these biopolymers for an $\mathrm{ABE}$ to simulate a mechanical biofilm removal.

Kawanisishi et al. stated requirements for an "artificial denture plaque" for denture care training as texture and color similar to the denture biofilm, resistance to removal under running water, possibility to dye with disclosing solutions, no risk for users, not affecting the training environment, and low environmental impact [15]. Texture and color have not been evaluated for the novel ABE. Except for one, all other requirements can be stated as given for the $\mathrm{ABE}$ independent from the mixing ratio. A minor influence on the training environment is given for $\mathrm{ABE}$ since the surface to be coated with $\mathrm{ABE}$ has to be sandblasted with silica-modified aluminum oxide.

Nevertheless, the resistance to brushing of the "artificial denture plaque" described by Kawanishi et al. has not been evaluated by a brushing simulator and thus cannot be directly compared with ABE prepared in this study [15].

The novel ABE developed in this pilot study allows an adjustment of its abrasion resistance to brushing from "very easy to remove" to "almost impossible to remove." Further studies are needed to adjust the abrasion resistance to that of natural oral biofilm. A specific area of a removable denture is cleaned with approximately 15 brushing strokes per 


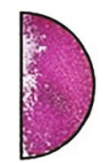

(a)

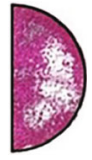

(b)

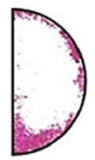

(c)

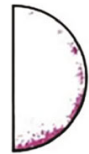

(d)

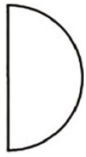

(e)

FIGURE 2: (a-e) Exemplary images of test specimen halves with dyed artificial biofilm equivalent (ABE, layer 2) in test series TS 3 for planimetric measurement of remaining $\mathrm{ABE}$ depending on brushing strokes numbers after brushing: (a) after 5 brushing strokes, (b) after 10 brushing strokes, (c) after 15 brushing strokes, (d) after 25 brushing strokes, and (e) after 45 brushing strokes. Black frames on the images of test specimen halves were added to original images for better visualization.

cleaning session [35]. For teeth, 10 to 15 brushing strokes per tooth are reported [36]. In this study, brushing strokes between 5 and 45 were chosen to determine the abrasion resistance of the ABE. Apart from the number of brushing strokes, the cleaning effect is also dependent on the contact weight of the brush head to the surface. Individual forces applied to manual toothbrushes during brushing cycles mainly range between $0.2 \mathrm{~N}$ and $4.5 \mathrm{~N}[32,36]$. For in vitro studies, conditions with 2 to $3 \mathrm{~N}$ are often used [36]. Thus, the standardized brushing experiments in this study were performed with a force of $2.9 \mathrm{~N}$, which corresponds to a weight of $300 \mathrm{~g}$.

An advantage of the novel ABE developed in this pilot study is the possibility to detect amounts of remaining $\mathrm{ABE}$ by staining with common plaque disclosing agents such as erythrosine. ChS and MC are polysaccharides and thus similar to the chemistry of the polysaccharides of the biofilm matrix. That may explain their affinity to plaque disclosing agents.

A limitation of the novel ABE developed in this pilot study is the absence of a microbial component. Thus, the $\mathrm{ABE}$ is not suitable to test antimicrobial oral care measures such as mouth rinses.

So far, the ABE was tested only on flat PMMA specimens. Further studies on curved specimens such as dentures are needed.

A further limitation of this study is the absence of toothpaste as the abrasiveness of the paste influence the brushing abrasion [36]. However, the use of toothpaste for the cleaning of denture bases is discussed controversially for its use may roughen the resin surfaces [37]. Tap water instead of distilled or deionized water was used in this study to simulate the real-life conditions as required, e.g., for denture care training. However, this might be considered a limitation of this study as standardization might be compromised and interactions between tap water ions and $\mathrm{ABE}$ cannot be excluded. To obtain standardization within the experiments, tap water from one source only was used.

The CPM was chosen to evaluate the amounts of $\mathrm{ABE}$ removal during brushing. This method is stated as objective and reliable and shows a high level of standardization and the ability to detect small changes in biofilm removal [28]. Nevertheless, the CPM is also a limiting factor in this study for this method is based on two-dimensional images and it is not possible to evaluate the changes in the thickness of a biofilm or the ABE until completely removed. Furthermore, the color intensity of the dyed $\mathrm{ABE}$ changes with increasing brushing strokes. This requires an exact selection of the color ranges for each image and thus is time-consuming.
Finally, the novel ABE described in this pilot study can be produced from inexpensive, nonhazardous, eco-friendly materials using simple, affordable, user-friendly laboratory techniques common to dental professionals.

It is a promising approach that should be further developed and investigated especially in terms of adjusting the mechanical properties such as abrasion to brushing from the $\mathrm{ABE}$ to natural denture biofilm.

\section{Conclusions}

In this in vitro pilot study, a novel biopolymer-based $\mathrm{ABE}$ was developed and its abrasion resistance was tested using automatic brushing with toothbrushes. The ABE allowed control of its abrasion resistance by changing the ratio of the two ingredients ChS and MC. Abrasion resistance adjustment covered a range from "very easy to remove" with ChS in low ratio to "almost impossible to remove" with ChS in high ratio. An undergoing study investigates the abrasion resistance of naturally grown biofilms on PMMA denture bases' surfaces to allow for the adaptation of the ABE abrasion resistance as close as possible to that of natural biofilms.

\section{Data Availability}

The data used to support the findings of this study are included within the article. The complete dataset is available by the corresponding author on request.

\section{Disclosure}

This research was performed as part of the doctoral thesis and as a part of employment of the authors at Department of Prosthetic Dentistry, Carl Gustav Carus Faculty of Medicine, Technische Universität Dresden.

\section{Conflicts of Interest}

The authors declare that there is no conflict of interest regarding the publication of this article.

\section{Authors' Contributions}

Katarzyna Kresse-Walczak, Heike Meissner, and Klaus Boening were involved in study design, interpretation of data, revising the article, and final approval. Jan-Philipp Kunze was involved in acquisition, analysis, interpretation of data, wrote the article draft, and approved the final version. 


\section{References}

[1] F. Müller, M. Naharro, and G. E. Carlsson, "What are the prevalence and incidence of tooth loss in the adult and elderly population in Europe?," Clinical Oral Implants Research, vol. 18, pp. 2-14, 2007.

[2] the DMS V Surveillance Investigators' Group, R. A. Jordan, C. Bodechtel et al., "The fifth German oral health study (fünfte deutsche mundgesundheitsstudie, DMS V) - rationale, design, and methods," BMC Oral Health, vol. 14, no. 1, p. 161, 2014.

[3] L. Gendreau and Z. G. Loewy, "Epidemiology and etiology of denture stomatitis," Journal of Prosthodontics, vol. 20, no. 4, pp. 251-260, 2011.

[4] G. Ramage, L. O’Donnell, L. Sherry et al., "Impact of frequency of denture cleaning on microbial and clinical parameters-a bench to chairside approach," Journal of Oral Microbiology, vol. 11, no. 1, p. 1538437, 2019.

[5] J. Barochia and S. Kamath, "Evaluation of the effect of denture cleansers on the surface roughness of hard denture base material: an in vitro study," Indian Journal of Dental Research, vol. 29, no. 5, pp. 657-662, 2018.

[6] K. V. Martins and S. M. . L. Gontijo, "Tratamento da estomatite protética: revisão de literatura," Revista Brasileira de Odontologia, vol. 74, no. 3, pp. 215-220, 2017.

[7] S. Susewind, R. Lang, and S. Hahnel, "Biofilm formation and Candida albicans morphology on the surface of denture base materials," Mycoses, vol. 58, no. 12, pp. 719-727, 2015.

[8] K. Hama, Y. Iwasa, Y. Ohara et al., "Pneumonia incidence and oral health management by dental hygienists in long-term care facilities: a 1-year prospective multicentre cohort study," Gerodontology, pp. 1-10, 2021.

[9] C. D. van der Maarel-Wierink, J. N. Vanobbergen, E. M. Bronkhorst, J. M. G. A. Schols, and C. de Baat, "Oral health care and aspiration pneumonia in frail older people: a systematic literature review," Gerodontology, vol. 30, no. 1, pp. 3-9, 2013.

[10] P. di Martino and Groupe biofilm et comportement microbien aux interfaces, Laboratoire ERRMECe-EA1391, Université de Cergy-Pontoise, rue Descartes site de Neuville-sur-Oise 95031 Cergy-Pontoise, cedex France, "Extracellular polymeric substances, a key element in understanding biofilm phenotype," AIMS microbiology, vol. 4, no. 2, pp. 274-288, 2018.

[11] N. S. Jakubovics, S. D. Goodman, L. Mashburn-Warren, G. P. Stafford, and F. Cieplik, "The dental plaque biofilm matrix," Periodontology 2000, vol. 86, no. 1, pp. 32-56, 2021.

[12] H.-C. Flemming and J. Wingender, "Relevance of microbial extracellular polymeric substances (EPSs)-part I: structural and ecological aspects," Water Science and Technology, vol. 43 , no. 6 , pp. $1-8,2001$.

[13] D. W. Volpenhein, M. E. Walsh, P. A. Dellerman, and T. A. Burkett, "A new method for in vitro evaluation of the interproximal penetration of manual toothbrushes," The Journal of Clinical Dentistry, vol. 5, no. 1, pp. 27-33, 1994.

[14] C. P. Ernst, B. Willershausen, G. Driesen, P. R. Warren, and P. Hilfinger, "A robot system for evaluating plaque removal efficiency of toothbrushes in vitro," Quintessence International, vol. 28, no. 7, pp. 441-445, 1997.

[15] K. Kawanishi, T. Okahashi, H. Aita et al., "Usefulness of the newly developed artificial denture plaque for practical denture care training," Clinical and experimental dental research, vol. 6 , no. 2, pp. 254-265, 2020.
[16] H. Lamfon, Z. Al-Karaawi, M. McCullough, S. R. Porter, and J. Pratten, "Composition of in vitro denture plaque biofilms and susceptibility to antifungals," FEMS Microbiology Letters, vol. 242, no. 2, pp. 345-351, 2005.

[17] T. Coenye, K. de Prijck, B. de Wever, and H. J. Nelis, "Use of the modified Robbins device to study the in vitro biofilm removal efficacy of NitrAdine ${ }^{\mathrm{TM}}$, a novel disinfecting formula for the maintenance of oral medical devices," Journal of Applied Microbiology, vol. 105, no. 3, pp. 733-740, 2008.

[18] H. F. Oliveira Paranhos, C. H. Silva-Lovato, R. F. De Souza et al., "Effect of three methods for cleaning dentures on biofilms formed in vitro on acrylic resin," Journal of Prosthodontics, vol. 18, no. 5, pp. 427-431, 2009.

[19] M. Wieckiewicz, E. Wolf, G. Richter, H. Meissner, and K. Boening, "New concept of polymethyl methacrylate (PMMA) and polyethylene terephthalate (PET) surface coating by chitosan," Polymers, vol. 8, no. 4, p. 132, 2016.

[20] I. Younes and M. Rinaudo, "Chitin and chitosan preparation from marine sources. Structure, properties and applications," Marine Drugs, vol. 13, no. 3, pp. 1133-1174, 2015.

[21] M. N. R. Kumar, "A review of chitin and chitosan applications," Reactive and Functional Polymers, vol. 46, no. 1, pp. 1-27, 2000.

[22] K.-G. Rosell, "Distribution of substituents in methylcellulose," Journal of Carbohydrate Chemistry, vol. 7, no. 3, pp. 525-536, 1988.

[23] J. Arboleya and P. Wilde, "Competitive adsorption of proteins with methylcellulose and hydroxypropyl methylcellulose," Food Hydrocolloids, vol. 19, no. 3, pp. 485-491, 2005.

[24] P. L. Nasatto, F. Pignon, J. L. Silveira, M. Duarte, M. Noseda, and M. Rinaudo, "Interfacial properties of methylcelluloses: the influence of molar mass," Polymers, vol. 6, no. 12, pp. 2961-2973, 2014.

[25] I. G. Donhowe and O. Fennema, "The effects of plasticizers on crystallinity, permeability, and mechanical properties of methylcellulose films," Journal of Food Processing \& Preservation, vol. 17, no. 4, pp. 247-257, 1993.

[26] M.-C. Chen, G. H.-C. Yeh, and B.-H. Chiang, "Antimicrobial and physicochemical properties of methylcellulose and chitosan films containing a preservative," Journal of Food Processing \& Preservation, vol. 20, no. 5, pp. 379-390, 1996.

[27] V. S. Kumari, S. K. Basha, and P. Sudha, "Physicochemical and morphological evaluation of chitosan/poly (vinyl alcohol)/ methylcellulose chemically cross-linked ternary blends," Polymer Bulletin, vol. 68, no. 5, pp. 1387-1393, 2012.

[28] A. Al Jaghsi, T. Mundt, and R. Biffar, "Reproducibility of a new computerized planimetric method for the measurement and assessment of removable dental prostheses plaque," The International Journal of Prosthodontics, vol. 30, no. 4, pp. 377-383, 2017.

[29] H.-C. Flemming and J. Wingender, "The biofilm matrix," Nature Reviews. Microbiology, vol. 8, no. 9, pp. 623-633, 2010.

[30] T. Sterzenbach, R. Helbig, C. Hannig, and M. Hannig, "Bioadhesion in the oral cavity and approaches for biofilm management by surface modifications," Clinical Oral Investigations, vol. 24, no. 12, pp. 4237-4260, 2020.

[31] R. G. Ledder, J. Latimer, S. Forbes, J. L. Penney, P. K. Sreenivasan, and A. J. McBain, "Visualization and quantification of the oral hygiene effects of brushing, dentifrice use, and brush wear using a tooth brushing simulator," Frontiers in Public Health, vol. 7, no. 91, 2019. 
[32] F. M. Sander, C. Sander, and F. G. Sander, "Dental care with manual toothbrushes during fixed orthodontic treatment-a new testing procedure," Journal of Orofacial Orthopedics, vol. 66, no. 4, pp. 299-306, 2005.

[33] D. Lazarescu, S. Boccaneala, A. Illiescu, and J. A. De Boever, "Efficacy of plaque removal and learning effect of a powered and a manual toothbrush," Journal of Clinical Periodontology, vol. 30, no. 8, pp. 726-731, 2003.

[34] T. Lang, S. Staufer, B. Jennes, and P. Gaengler, "Clinical validation of robot simulation of toothbrushing-comparative plaque removal efficacy," BMC Oral Health, vol. 14, no. 1, pp. 1-9, 2014.

[35] J. C. Sexson and R. W. Phillips, "Studies on the effects of abrasives on acrylic resins," The Journal of Prosthetic Dentistry, vol. 1, no. 4, pp. 454-471, 1951.

[36] A. Wiegand and T. Attin, "Design of erosion/abrasion studiesinsights and rational concepts," Caries Research, vol. 45, no. 1, pp. 53-59, 2011.

[37] Y. H. Chang, C. Y. Lee, M. S. Hsu, C. K. K. Du JK, and J. H. $\mathrm{Wu}$, "Effect of toothbrush/dentifrice abrasion on weight variation, surface roughness, surface morphology and hardness of conventional and CAD/CAM denture base materials," Dental Materials Journal, vol. 40, no. 1, pp. 220-227, 2021. 